\title{
Performance of Chemiluminiscence Assay using Reverse Algorithm for Syphilis Screening in Blood Donors
}

\author{
Mamata Kale ${ }^{1}$, Subhashish Das ${ }^{2 *}$, Mahesh Venkatesha ${ }^{3}$ and Parvangada Madappa \\ Beena $^{1}$ \\ ${ }^{1}$ Department of Microbiology, Sri Devraj Urs Medical College, Tamaka, Kolar, Karnataka, India. ${ }^{2}$ Department of \\ Pathology, Sri Devraj Urs Medical College, Tamaka, Kolar, Karnataka, India. ${ }^{3}$ Department of Community Medicine, \\ Chamarajanagar Institute of Medical Sciences, Chamarajanagar, Karnataka, India.
}

\begin{abstract}
Better screening tests for syphilis are required to help ensure safer blood supply. Reverse screening for syphilis using specific test like chemiluminescence immunoassay (CLIA) allows automation for high sample load testing, followed by testing of positive sera with a non-treponemal. In case of discordant result between the two tests, the sample is tested with another confirmatory specific test. The objective of this study was to evaluate the performance of automated Vitros Syphilis Treponema pallidum antibody chemiluminescence immunoassay as a screening test in blood donors using reverse algorithm for testing syphilis. A total of 16362 blood donors were screened for syphilis by CLIA. The CLIA positive samples and 100 CLIA negative samples were further tested by Rapid Plasma Reagin Test (RPR) and Treponema pallidum haemagglutination test (TPHA). 155 (0.95\%) sera were positive by CLIA among which $34(21.93 \%)$ were reactive by RPR and $32(20.6 \%)$ were confirmed positive by TPHA. $121(78.06 \%)$ samples yielded discordant result with RPR, of which $10(6.45 \%)$ were confirmed positive by TPHA. The sensitivity of CLIA in comparison to TPHA was $100 \%$, specificity $46.95 \%$, positive predictive value $27.1 \%$, negative predictive value $100 \%$ and kappa coefficient 0.2257 (poor agreement). CLIA detected $10(6.45 \%)$ cases of syphilis which would have been missed if RPR was used as the initial screening test. CLIA can be employed for screening syphilis in blood donors for its good sensitivity, ease of automation and short turn-around-time.
\end{abstract}

Keywords: Chemiluminiscenscence assay, syphilis, reverse algorithm, blood donors.

*Correspondence: daspathology@gmail.com; +919480849818

(Received: 10 August 2018; accepted: 14 October 2018)

Citation: Mamata Kale, Subhashish Das, Mahesh Venkatesha and Parvangada Madappa Beena, Performance of Chemiluminiscence Assay using Reverse Algorithm for Syphilis Screening in Blood Donors, J Pure Appl Microbiol., 2018; 12(4):2253-2257. http:// dx.doi.org/10.22207/JPAM.12.4.65

(c) The Author(s) 2018. Open Access. This article is distributed under the terms of the Creative Commons Attribution 4.0 International License which permits unrestricted use, sharing, distribution, and reproduction in any medium, provided you give appropriate credit to the original author(s) and the source, provide a link to the Creative Commons license, and indicate if changes were made. 


\section{INTRODUCTION}

Syphilis a sexually transmitted infection is caused by the spirochete Treponema pallidum subsp. pallidum. The disease is chronic and progresses through distinct clinical stages and diverse clinical manifestations making laboratory testing a very important aspect for detecting syphilis. Serology remains the mainstay for laboratory diagnosis of syphilis as $T$. pallidum cannot be stained or cultured with routine laboratory methods. Serological tests for syphilis fall into two categories: non treponemal tests and treponemal tests. Non-treponemal tests includes tests like Venereal Disease Research Laboratory (VDRL) and the Rapid Plasma Reagin Test (RPR) that identifies antilipoidal antibodies produced by the host in response to lipoidal materials released from damaged host cells and lipid present on the cell surface of T. pallidum. The treponemal tests detect antibodies specific to T. pallidum and include fluorescent Treponemal antibody absorption (FTA-ABS), T. pallidum haemagglutination test (TPHA), and T. pallidum particle agglutination (TPPA). ${ }^{1,2}$

According to the Drug and Cosmetics Act and Rules 1945, the blood banks may choose testing of donor samples for syphilis with a nontreponemal test or TPHA. ${ }^{3}$ In India, RPR is the most commonly used test for screening blood donors. ${ }^{2,4}$ However, screening with RPR may not detect all cases of syphilis as non-treponemal tests lack sensitivity in few stages of syphilis like early primary syphilis and late latent syphilis. A false negative result may also be due to the prozone phenomenon. In addition, biological false positive reports are seen in a variety of clinical conditions.

Recently, completely automated chemiluminiscence immunoassays (CLIA) which use recombinant Treponemal antigen to identify IgG and IgM are available and are becoming the method of choice in many establishments for the reliable detection of $T$. pallidum infections, facilitating clear, consistent, and more objective recording of results. Automation of the test will also reduce the time and labour required for screening syphilis if routinely emplyoyed. ${ }^{4,5}$

Reverse sequence screening algorithm is provided by the Centers for Disease Control and Prevention $(C D C)$ in which a specific treponemal test like ELISA/CLIA is performed first, followed by testing reactive sera with non treponemal test like RPR or VDRL. In case of discordance between the two tests, another confirmatory specific treponemal test is performed. ${ }^{6}$

The performance of Vitros Syphilis Treponema Pallidum Antibody (TPA) chemiluminescence immunoassay has been previously evaluated with routine clinical samples and also blood donor samples. ${ }^{4,7}$ However, there are no publications on the yield of reverse algorithm in blood bank settings. The purpose of this study was to evaluate Vitros Syphilis Treponema pallidum antibody chemiluminescence immunoassay (CLIA) as screening test in blood donors using reverse algorithm for testing syphilis.

\section{MATERIALS AND METHODS}

The study was conducted from June 2015 to May 2017 at R L Jalappa Hospital and Research Centre, Kolar. A total of 16362 blood donors were screened for syphilis by Vitros Syphilis Treponema Pallidum Antibody (TPA) chemiluminescence immunoassay (Ortho Clinical Diagnostics, Inc., High Wycombe, United Kingdom). The Vitros Syphilis TPA assay is a qualitative assay that detects total antibodies (IgG, IgM and IgA) to recombinant Treponema pallidum (TP) antigens TP15, TP17, TP47. The antibodies in the serum reacts with biotinylated and horseradish peroxidase (HRP)-labelled TP antigen conjugate. The antigen-antibody complex is captured by streptavidin-coated wells. The bound HRP conjugate is measured by a luminescent reaction. The bound HRP conjugate is directly proportional to the concentration of anti-TP antibody. The cut off value is obtained from the calibrator. Sample result is calculated by the ratio of signal of test sample/cut off value (S/C). The ratio of $<0.8$ were considered negative, between $\geq 0.8$ and $\leq 1.2$ as borderline and ratio $\geq 1.2$ as positive. The betweenrun precision of the CLIA was performed using positive and negative controls, run once a day during the study period.

All the CLIA positive and 100 CLIA negative samples were further tested by RPR (Span Diagnostics Ltd., India) and TPHA (Omega Diagnostic, Scotland) as per the manufacturer instructions. TPHA was considered as gold standard test for comparison of results. Specimen 
that were tested negative by all the three methods were considered definite negative; CLIA positive specimen that were non-reactive by RPR as discordant and both CLIA and TPHA positive were considered confirmed positive.

Data was entered into Microsoft excel data sheet and was analyzed using SPSS 22 version software. Categorical data was represented in the form of frequencies and proportions. Continuous data was represented as mean and standard deviation. Correlation between CLIA and TPHA was determined by kappa coefficient.

\section{RESULTS}

A total of 155 (0.95\%) out of 16362 voluntary blood donors were screened positive for syphilis by CLIA. Mean age of reactive donors was $32.93 \pm 7.336$ years and majority were

Table 1. Comparison of CLIA results with RPR and TPHA $(n=255)$

\begin{tabular}{lcclcc}
\hline CLIA & \multicolumn{2}{c}{ RPR } & & \multicolumn{2}{c}{ TPHA } \\
\cline { 2 - 3 } \cline { 5 - 6 } & Reactive & $\begin{array}{c}\text { Non- } \\
\text { reactive }\end{array}$ & & Positive & Negative \\
\hline Positive & 34 & 121 & & 42 & 113 \\
Negative & 2 & 98 & & 0 & 100 \\
Total & 36 & 219 & & 42 & 213 \\
\hline
\end{tabular}

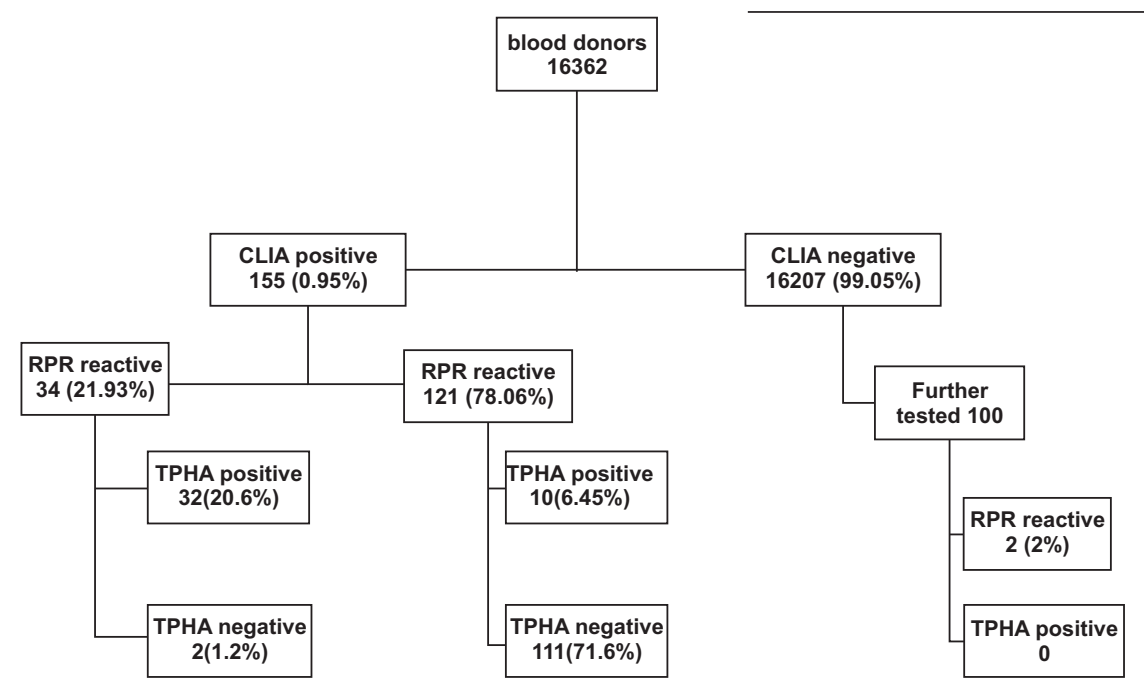

males 142(91.6\%). Among the 155 CLIA positive, $34(21.93 \%)$ were reactive by RPR of which 32 (20.64\%) were confirmed positive by TPHA (fig 1). 121 (78.06\%) samples were discordant (RPR non-reactive), among which 10 (6.45\%) were confirmed positive by TPHA. A total of 111(71.6\%) were negative by both RPR and TPHA. Among the 100 CLIA negative samples, RPR yielded 2(2\%) biological false reactive samples which were negative by TPHA.

Table 1 shows comparison of CLIA with RPR and TPHA. The performance of CLIA with TPHA is shown in table 2. CLIA had a sensitivity of $100 \%$ ( $95 \% \mathrm{Cl}, 91.62$ to 100$)$, specificity of $46.95 \%$ (95\% $\mathrm{Cl}, 40.36$ to 53.65$)$, and the diagnostic accuracy of $55.69 \%$ (95\% Cl, 49.55 to 61.65$)$. The agreement between CLIA and TPHA was poor ( $k=0.22$; $95 \%$ $\mathrm{Cl}, 0.14-0.30)$.

Table 2. Performance of CLIA in comparison with TPHA

\begin{tabular}{|c|c|c|}
\hline Parameter & Estimate & $\begin{array}{l}\text { 95\% Confidence } \\
\text { interval }\end{array}$ \\
\hline Sensitivity & $100 \%$ & 91.62 - 100 \\
\hline Specificity & $46.95 \%$ & $40.36-53.65$ \\
\hline Positive & & \\
\hline $\begin{array}{l}\text { Predictive Value } \\
\text { Negative Predictive }\end{array}$ & $27.1 \%$ & $20.72-34.58$ \\
\hline Value & $100 \%$ & $96.3-100$ \\
\hline $\begin{array}{l}\text { Diagnostic Accuracy } \\
\text { Cohen's kappa }\end{array}$ & $55.69 \%$ & $49.55-61.65$ \\
\hline (Unweighted) & 0.2257 & $0.148-0.3034$ \\
\hline
\end{tabular}

Fig. 1. Reverse Algorithm for blood donor screening for syphilis. 


\section{DISCUSSION}

Chemiluminiscence assays are employed for reverse sequence screening of syphilis for ease of automation especially in high sample load testing laboratories. In the blood bank settings, the automation of the test along with testing of other transfusion transmitted infections like anti-HIV, $\mathrm{HbsAg}$ and anti-HCV provides rapid results and improves workflow with optimal release of blood. The present study has evaluated the performance of Vitros ECi Syphilis Treponema Pallidum antibody chemiluminescence immunoassay as a screening test for syphilis in blood donors using the reverse algorithm. The results of the assay were compared with RPR and TPHA.

The sensitivity of the Vitros CLIA was $100 \%$, which is comparable with sensitivity of other studies. But the specificity varied largely; $46.94 \%$ in comparison with $99.65 \%$ and $100 \%$ in other studies. ${ }^{4,7}$ Since TPHA, the confirmatory test was performed only with 100 CLIA negative samples due to resource constraints the specificity rate, the diagnostic accuracy, and kappa value may be lower.

The discordant result between CLIA and RPR was seen in 121 (78.06\%), among which 10 (6.45\%) were confirmed positive by TPHA. The 10 (6.45\%) cases of syphilis detected by CLIA would have been missed if RPR was the initial screening test. These cases could be latent untreated syphilis or past treated syphilis. In a study by Gatrix et al., the proportion of discordant late latent syphilis increased significantly after the introduction of reverse screening algorithm. These patients were identified as high risk patients by their health care providers and were treated. ${ }^{8}$ The sensitivity of RPR is lower than that of treponemal tests, especially in primary and late latent syphilis. A non-treponemal test like RPR is recommended in screening blood donor population where the incidence and prevalence of syphilis are high. In high prevalent population, the risk of transmission cannot be reduced through donor selection strategies and screening is done only to identify the high-risk donors with evidence of recent infection. ${ }^{2}$

111 (71.6\%) discordant sera among 155 CLIA positive samples were negative by confirmatory TPHA test in the reverse algorithm. A CDC evaluation of reverse screening algorithm in four New York City laboratories showed that percentage of nonreactive confirmatory treponemal tests was 31.6 and the reverse algorithm yielded $2.9 \%$ higher false positive results in low prevalence population compared to high prevalence population. ${ }^{6}$ The percentage of false positive in the present study could be higher due to low prevalence of the disease in the population. The reported prevalence of syphilis in the area is $1.18 \%$ in out-patients and inpatients and $0.35 \%$ among blood donors. ${ }^{9,10}$

The obtained false positive result 113 (0.69\%) out of 16362 totally screened is similar to the study by CDC, where $0.61 \%$ ( 866 out of 140176 screened) were false positive. ${ }^{6}$ The result is also comparable with another study by Morgani A et al. where $1.4 \%$ (129 out of 9210) of the sera submitted for routine screening and samples from blood donors were false positive by CLIA. ${ }^{11}$ The limitation of many serological tests is false negative results in early syphilis, during which the titres of antibodies against T. pallidum can be low and hard to detect. ${ }^{12}$ Early detection of syphilis is important to prevent further transmission of syphilis and to provide timely treatment. As CLIA identifies both IgM and IgG antibodies, these probably could be cases of primary syphilis. CLIA also has good sensitivity in detection of early seroconversion of syphilis. ${ }^{13,14}$

This study had few limitations. Further clinical evaluation of CLIA positive cases was not done and the performance of the test according to the stage of syphilis could not be assessed. Since RPR and TPHA were performed only on CLIA positive sera and 100 CLIA negative sera, the tests for comparison could not be appropriately interpreted.

Despite selection of low risk donors, the risk of transmission persists to blood and blood product recipients if screening test lacks sensitivity. The reverse screening algorithm detected 10 cases of possible latent syphilis, though the false positive was higher. Our finding support prior data suggesting that reverse screening may enhance the sensitivity for detection of early or latent syphilis.

\section{CONCLUSION}

The Vitros TPA assay is highly sensitive assay and considering its suitability to automation CLIA can be employed as a screening test for syphilis in blood bank and routine clinical 
laboratory. However further confirmation by other treponemal test like TPHA is required.

\section{REFERENCES}

1. Larsen S A, Steiner B M and Rudolph AH. Laboratory diagnosis and interpretation of tests for syphilis. Clin. Microbiol Rev. 1995; 8(1):1-21.

2. Kaur G. and Kaur P. Syphilis testing in blood donors: an update. Blood Transfusion. 2015; 13:197-204.

3. Drugs and Cosmetic Act. Available from http:// www.cdsco.nic.in/writereaddata/Drugs and CosmeticAct.pdf.

4. Tiwari AK, Pandey PK, Dara RC, Rawat GS, Raina V, Bhargava R. Evaluation of a new serological test for syphilis based on chemiluminescence assay in a tertiary care hospital. Asian J Transfus Sci. 2015; 9(1):65-9.

5. Park $Y$, Park $Y$, Young Joo S, Park MH, Kim HS. Evaluation of a Fully Automated Treponemal Test and Comparison with conventional VDRL and FTA-ABS tests. Am J Clin Pathol. 2011; 136:705710.

6. Centers for Disease Control and Prevention (CDC). 2011. Discordant results from reverse sequence syphilis screening - Five laboratories, United States, 2006-2010. MMWR Morb Mortal Wkly Rep. 2011; 60:133-7.

7. González V, Fernández G, Dopico E, Margall N, Esperalba J, Muñoz C, Castro E, Sulleiro E, Matas L. Evaluation of the Vitros Syphilis TPA chemiluminescence immunoassay as a firstline method for reverse syphilis screening. J
Clin Microbiol. 2015; 53:1361-4. doi:10.1128/ JCM.00078-15.

8. Gatrix J, Plitt S, Lee BE, Ferron L, Anderson $B$, Verity $B$ et. al. Impact of reverse sequence screening on new diagnoses of late latent syphilis in Edmonton, Canada. Sexually transmitted dis. 2012; 39(7): 528-30.

9. Archana B R, Prasad S R, Beena P M, Okade R. Letters to Editor Making serological diagnosis of syphilis more accurate. Ind J of Sexually Transm Dis and AIDS. 2014; 35(1): 70-71.

10. Das S, HarendraKumar HL. Seroprevalence of syphilis among voluntary blood Donors: an institutional study. International J Basic and App Med Sci 2014; 4(1): 198-203.

11. Marangoni A, Moroni A, Accardo S, Cevenini R. Laboratory diagnosis of syphilis with automated assays. J Clin Lab Analysis. 2009; 23: 1-6.

12. Knight CS, Crum MA, Hardy RW. Evaluation of the LIAISON chemiluminescence immunoassay for diagnosis of syphilis. Clin Vaccine Immunol. 2007; 14: 710-713.

13. Kremastinou J, Polymerou V, Lavranos D, Aranda Arrufat A, Harwood J, Martínez Lorenzo MJ, Ng KP, Queiros L, Vereb I, Cusini M. Evaluation of Elecsys Syphilis assay for routine and blood screening and detection of early infection. J Clin Microbiol. 2016; 54: 2330 -6.

14. Young H, Pryde J, Duncan L, Dave J. The architect syphilis assay for antibodies to Treponema pallidum: An automated screening assay with high sensitivity in primary syphilis. Sex Transm Infect. 2009; 85:19-23. 